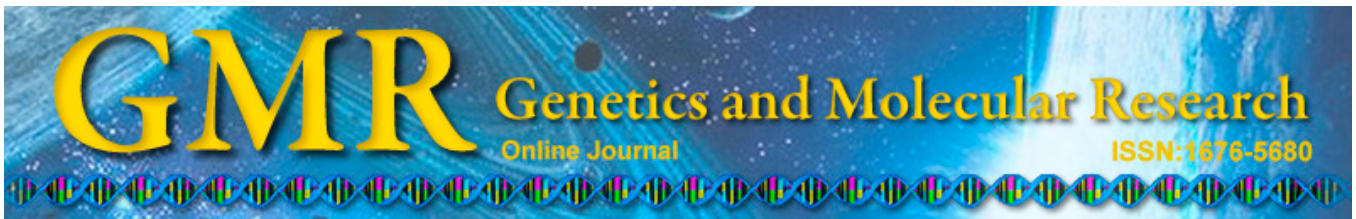

\title{
Cloning and analysis of the soybean MEKK gene
}

\author{
A.-H. Sha ${ }^{1,2}$, H.-P. Ba ${ }^{1}$, Z.-H. Shan ${ }^{1}$, H.-F. Chen ${ }^{1}$, S.-L. Chen ${ }^{1}$, D.-Z. Qiu', \\ X.-A. Zhou ${ }^{1}$ and Y.-H. Chen ${ }^{3}$
}

${ }^{1}$ Oil Crops Research Institute of the Chinese Academy of Agricultural Sciences, Key Laboratory of Oil Crop Biology of the Ministry of Agriculture, Wuhan, China ${ }^{2}$ Agriculture College of Yangtze University,

Hubei Collaborative Innovation Center for Grain Industry, Jingzhou, China

${ }^{3}$ College of Agriculture, Hainan University, Haikou, China

Corresponding author: X.-A. Zhou

E-mail:xinan_zhou@163.com

Genet. Mol. Res. 14 (2): 3625-3632 (2015)

Received June 9, 2014

Accepted October 27, 2014

Published April 17, 2015

DOI http://dx.doi.org/10.4238/2015.April.17.12

\begin{abstract}
In this paper, homologous cloning methods were used to clone the soybean $G m M E K K$ gene, which possesses a high degree of similarity to Arabidopsis thaliana AtMEKK1. AtMEKK1 is formed by 595 amino acids, and its secondary structure is formed by 38 irregular curls, $24 \alpha$ helix, $14 \beta$, with S-TKc domain, transmembrane domain and does not have membrane spanning domain and signal peptide. GmMEKKGFP subcellular localization fusion and prokaryotic expression vectors were generated and it was revealed that GmMEKK encodes a highly conserved $66.8-\mathrm{kDa}$ nuclear protein that is expressed in soybean roots, stem floral pieces, and leaves. A real-time quantitative PCR analysis of GmMEKK under different abiotic stresses revealed that the expression level of GmMEKK increased under drought and low phosphorus and nitrogen conditions. Taken together, these data suggest that GmMEKK may play an important role in the soybean abiotic stress response.
\end{abstract}

Key words: Mitogen-activated protein kinase; Subcellular localization; Serine/threonine protein kinase; Real-time fluorescent quantitative PCR; Semi-quantitative PCR 


\section{INTRODUCTION}

The mitogen-activated protein kinase (MAPK) signal transduction pathway is involved in a variety of biological and abiotic stress responses such as the hormone response, cell division and differentiation, disease resistance, and plant growth (Hirofumi et al., 2006; Suarez-Rodriguez et al., 2007). This process is formed by a level 3 phosphorylation enzymatic cascade that includes mitogen-activated protein kinase kinase kinases (MAPKKKs; MAP3K), mitogen-activated protein kinase kinases (MAPKKs; MAP2K), and mitogen-activated protein kinases (MAPKs). As the substrates are phosphorylated, the upstream signal cascade is amplified and passed down to effector molecules. MEKK1 is an important part of the signal transduction pathway as it is responsible for receiving the extracellular signal from the membrane receptor. Upon activation, MEKK1 transfer the extracellular stimulation signal from cells to the nucleus through the phosphorylation of a downstream gene, causing a cell biology response (Xu et al., 2005). While more than 60 MAPKKKs have been cloned in Arabidopsis (Suarez-Rodriguez et al., 2007), further studies on the cloning and function of MAPKKK genes in main crops are required. Drought, low nitrogen and phosphorus levels, and other environmental stresses seriously affect crop growth, development, and yield. In response to these stresses, crops have evolved a series of physiological stress response defense systems that incorporate cellular metabolism, receiving and imparting environmental signals, and the activation of specific subset of genes to produce an appropriate physiological response. Stressinduced signal transduction is a three-dimensional network with the MAPK signaling cascade in the network center. Through a series of phosphorylation and dephosphorylation events, the cascade will induce a large variety of stress signals, thereby regulating gene expression.

In this study, the soybean gene $G m M E K K$ was cloned. It is highly homologous with AtMEKK1 and bioinformatic and subcellular localization analyses suggest that GmMEKK and AtMEKK may share similar biological functions. At low nitrogen, phosphorus, and drought stress analyses of GmMEKK expression in different periods, we found that GmMEKK might be involved in the process of phosphorylation and dephosphorylation in soybean. As $M E K K$ is implicated in the adaptive response of soybean to environmental adversity, it lays a foundation for further research on the function of GmMEKKs in soybean.

\section{MATERIAL AND METHODS}

\section{Materials}

The plant materials in this study are Zhongdou No. 29 (ZD29), Tianlong No. 1 (TL1), and Zhongdou No. 32 (ZD32). The breeding varieties are material No. 116 (N116), which is resistant to low phosphorus levels, and material No. 54 (N54), which is resistant to both low nitrogen levels and drought. The subcellular localization carrier is pCX-DG, received from the ABRC (Arabidopsis in the Resource Center, http://abrc.osu.edu/). The prokaryotic expression carrier is pGEX-6p-1 (lab stock). The competent Escherichia coli strain DH5 alpha was used in the transformations. The Agrobacterium characteristic strain was GV3101 (lab stock). BL21 (DE3) was a gift from Dr. Lee, Department of Beijing Institute of Life Sciences. 


\section{Drugs and reagents}

The restriction endonucleases and T4 DNA ligase were purchased from Takara Biotechnology Co., Ltd. The fidelity enzymes and DNA mark were purchased from Beijing Transgen Biotech Corporation. IPTG, PMSF, and DTT were purchased from Merck, Germany. RNA reverse transcriptase and RNA enzyme inhibitors were purchased from Promega. The high-fidelity enzyme, 100-kDa protein mark, 5-kb DNA Ladder Marker, 2-kb DNA Ladder Marker, RNA extraction kit, and dNTPs were purchased from Beijing Transgen Biotech Corporation. SYBR GREEN MIX was purchased from TOYOBA Company. DNA extraction kit was purchased from Tektronix Company. TRIzol was purchased from Invitrogen. Primers were synthesized at Invitrogen. Sequencing was performed by SunBio Company.

\section{Material preparation and extraction of total RNA}

ZD29, TL1, ZD32, N116, and 1 N54 were covered by paper to sprout. ZD29, TL1, and ZD32 were planted in a small basin containing nutrient soil and vermiculite (2:1). N116 and N54 were cultured in the normal Hoagland nutrient solution. The control group (30) was cultured in standard Hoagland nutrient solution. The root samples were collected on days $0,3,6$, 9, and 12. N54 was planted in standard Hoagland nutrient solution, and after 15 days, 10 plants were placed on a clean filter paper in a $25^{\circ} \mathrm{C}$ light-care incubator, and the root samples selected after 0 and $10 \mathrm{~h}$. The remainders were treated as the control group. The growth conditions of the soybean were 28 to $30^{\circ} \mathrm{C} /$ (day/night), with an illumination time of $16 \mathrm{~h} / 8 \mathrm{~h}$ (day/night), and a relative humidity of $60 \%$. Tissues from root, stem, leaf, flower, and fruit of ZD29, TN1, and ZD32 were collected in the mature period. The RNA extraction protocol was performed in liquid nitrogen according to the manufacturer protocol.

\section{Complimentary DNA generation and GmMEKK cloning}

Total RNA $(2 \mu \mathrm{g})$ was used as starting material. First-strand cDNA was generated using oligo $(\mathrm{dT})_{18}$ primers and M-MLV reverse transcriptase according to the manufacturer protocol. Using the Arabidopsis MEKK1 gene At4g08500 cDNA sequence (http://www. arabidopsis.org/) as a reference, a homologous gene (Glyma13g02470) was found in the PhytozomeV7.0 database (http://www.phytozome.net/soybean.php). Primers were designed against the Glyma13g02470 cDNA sequence and expanded coding domain sequence (CDS). The following primers were constructed for cloning into the subcellular localization carrier: obhp01 (5'-gATGATGAACCACCTACCTC-3') and obhp02 (5'-GTGACAAGTTTAACCCCTTC-3'). The primers constructed for cloning into the prokaryotic expression vector were as follows: obhp03 (5'-cgGGATCCATGAACCACCTACCTCGAAT-3') and obhp04 (5'-cggaattcGTCGA CTAGTTGGAGGTGACAAGTTTAAC-3'). The GmMEKK semi-quantitative and quantitative fluorescence primers were as follows: obhp05 (5'-GGATGTACTGTGTTGGAG-3') and obhp06 (5'-AAGGAGATGTGGAACCAG-3'). The actin (accession number V00450.1) primers used were as follows: obhp07 (5'-CCATGAGCTTCCTGATGGAC-3') and obhp08 (5'-GTGGTCTCATGAATTCCAGC-3'). The products of the first-strand cDNA synthesis reaction were diluted 10 times and $5 \mu \mathrm{L}$ was used as a template to amplify the full-length cDNA using the obhp01 and obhp02 Glyma13g02470 primers. PCRs $(50 \mu \mathrm{L})$ contained: $5.0 \mu \mathrm{L} 10 \mathrm{X}$ Taq DNA polymerase buffer, $1.5 \mu \mathrm{L} 0.25 \mathrm{mM}$ dNTP, $0.5 \mu \mathrm{L} 5 \mathrm{U} / \mu \mathrm{L}$ Taq DNA polymerase 
(Hifi), $3 \mu \mathrm{L}$ of $10 \mu \mathrm{M}$ forward and reverse primers, $35 \mu \mathrm{L}$ ultrapure water, and $5 \mu \mathrm{L}$ cDNA template. PCR conditions were: 4 min denaturation at $94^{\circ} \mathrm{C}, 35$ cycles of $94^{\circ} \mathrm{C}$ for $40 \mathrm{~s}, 54^{\circ} \mathrm{C}$ for $30 \mathrm{~s}, 72^{\circ} \mathrm{C}$ for $2 \mathrm{~min}$, followed by a 10 -min final extension at $72^{\circ} \mathrm{C}$. Gel electrophoresis was performed to analyze the PCR products.

\section{Generating the subcellular localization carrier}

Agarose gel (1\%) electrophoresis was used to recover the PCR products. pCX-DG was digested with $X \mathrm{cmI}$ for $3 \mathrm{~h}$ at $37^{\circ} \mathrm{C}$ and recovered by agarose gel electrophoresis (1\%). The purified PCR products were ligated to the carrier of fragment by the TAKARA T4 DNA ligase. The ligation products were transformed into DH5 alpha competent $E$. coli, seeded on selective LB plates containing $50 \mathrm{mg} / \mathrm{L}$ kanamycin, and incubated overnight at $37^{\circ} \mathrm{C}$. Single colonies were picked and subject to PCR for identification. Positive colonies were selected, cultured, and sent to SunBio Company for sequencing.

\section{Onion epidermal cell infection}

The constructed subcellular localization carriers were transformed into GV3101 and incubated at $28^{\circ} \mathrm{C}$ for $36 \mathrm{~h}$. The obhp 03 and osah 491 primers were used to identify positive transformants by colony PCR. Positive plaques were selected and individually cultured in LB liquid medium containing $50 \mathrm{mg} / \mathrm{L}$ kanamycin overnight at $28^{\circ} \mathrm{C}$ until the $\mathrm{OD}_{600 \mathrm{~nm}}$ reached 0.8-1.0. Cultures were centrifuged at $4{ }^{\circ} \mathrm{C}$, then resuspended in sterile $\mathrm{ddH}_{2} \mathrm{O}$, and diluted to an $\mathrm{OD}_{600 \mathrm{~nm}}$ of 0.6. Fresh onion epidermis was torn and soaked in infection medium for $20 \mathrm{~min}$ and transferred to the solid MS medium for $24 \mathrm{~h}$ at $22^{\circ} \mathrm{C}$. Subcellular localization was observed using a motorized microscope (OLYMPUS, BX61).

\section{Microscopy analysis and image collection}

Green fluorescent protein (GFP) was observed at $488 \mathrm{~nm}$ by microscopy using a 10X objective lens. An empty pCX-DG vector was used as a control.

\section{Generation and identification of the prokaryotic expression vector}

The pGEX-6p-1 expression vector and recovered GmMEKK fragments were double digested by the Bam HI and SalI restriction enzymes for $3 \mathrm{~h}$ at $37^{\circ} \mathrm{C}$. Agarose gel electrophoresis $(1 \%)$ was used to separate the cut plasmid and cDNA fragments and a T4 DNA ligase was used in the overnight, $16^{\circ} \mathrm{C}$ ligation. Ligation products $(2 \mu \mathrm{L})$ were used to chemically transform competent $E$. coli DH5 alpha cells. Bacteriaum liquid-coating dish containing bacteria $(200 \mu \mathrm{L})$ was cultured in liquid media containing ampicillin $(100 \mathrm{mg} / \mathrm{L})$ overnight at $37^{\circ} \mathrm{C}$. Twenty-four randomly selected colonies were screened by PCR and positive clones used to inoculate LB liquid media containing ampicillin $(100 \mathrm{mg} / \mathrm{L})$. Cultures were incubated overnight at $37^{\circ} \mathrm{C}$ and the plasmids extracted according to the manufacturer protocol. Following a double-restriction enzyme digestion, plasmids were sequenced by the SunBio company. The freeze-thaw method was used to transfer the sequenced plasmids to the competent $E$. coli strain BL21 (DE3). Cultures $(200 \mu \mathrm{L})$ were seeded on LB-ampicillin $(100 \mathrm{mg} / \mathrm{L})$ plates and 12 single colonies selected for PCR screening. Positive colonies inoculated LB liquid media containing ampicillin $(100 \mathrm{mg} / \mathrm{L})$ and incubated overnight at $37^{\circ} \mathrm{C}$. 


\section{RESULTS}

\section{Cloning the target gene}

The complete 9223-bp Glyma 13g02470 gene (published in phytozome database) contains 8 exons and 7 introns and encodes a 1785-bp cDNA (Figure 1). Using the designed Glyma13g02470 primers, a 1.8-kb fragment was obtained from ZD29, TL1, and ZD32 cDNA (Figure 2). The obtained fragments shared similar sequences with respect to each other, however, they differed slightly from the published Glyma13g02470 sequence with the residue at positions 568 and 1358. Since the majority of the sequences were identical to the published gene, they were confirmed to be GmMEKK.

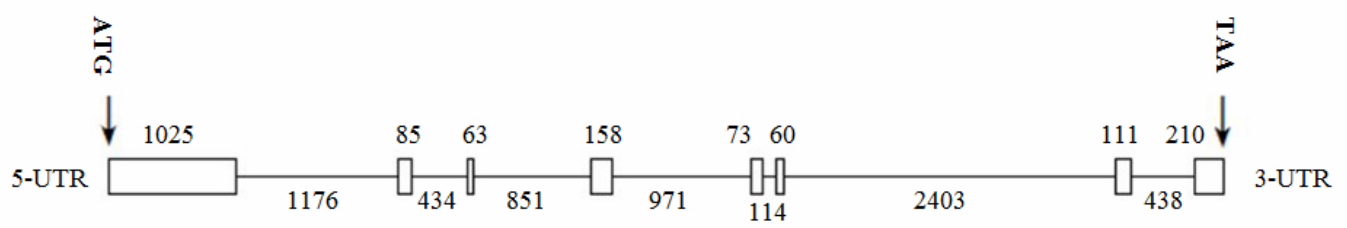

Figure 1. Gene structure of Glyma13g02470. Open rectangles and solid lines represent exons and introns, respectively. The image is drawn to scale with the unit being bp.

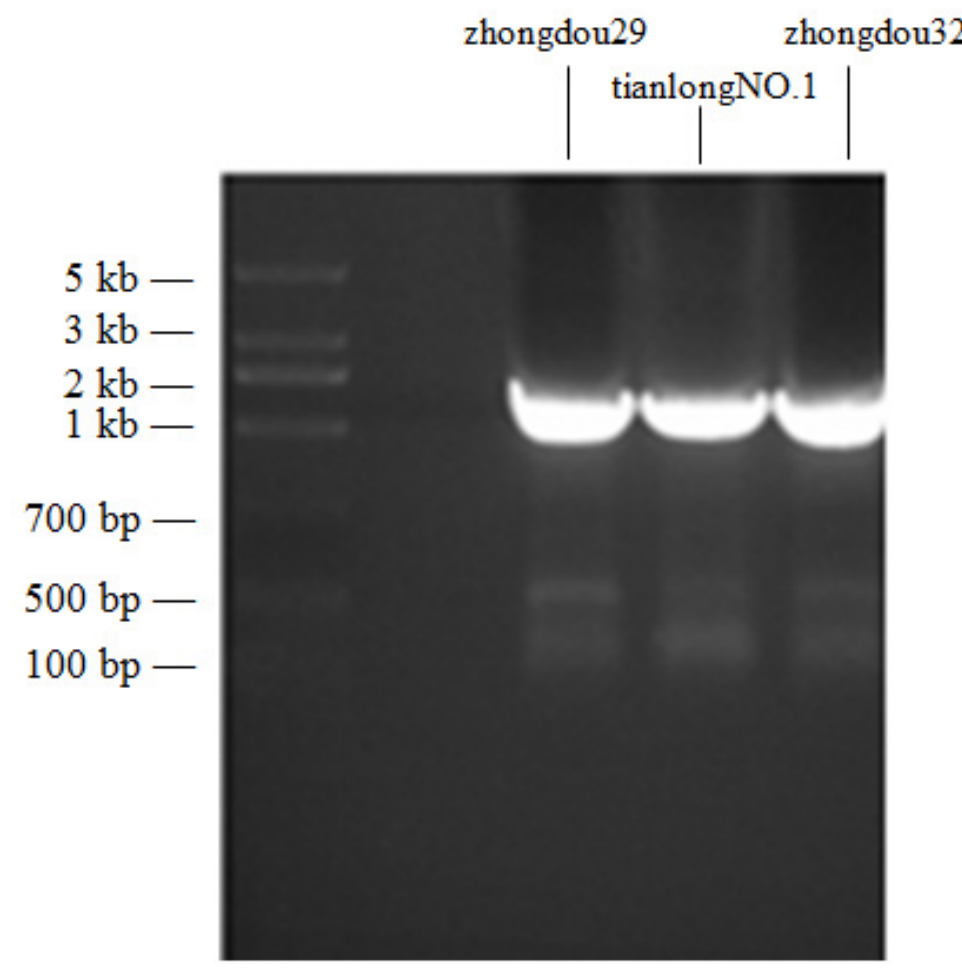

Figure 2. Amplification in the GmMEKK cDNA from Zhongdou 29, Tianlong No. 1, and Zhongdou 32 using the obhp01 and obhp02 primers. 


\section{Expression and detection of GmMEKK in E. coli}

The predicted sizes of GmMEKK and GFP-GmMEKK are 66.8 and $92.8 \mathrm{kDa}$, respectively (Figure 3). The induced GFP-GmMEKK protein was $93 \mathrm{kDa}$, indicating that it was indeed expressed in E. coli.

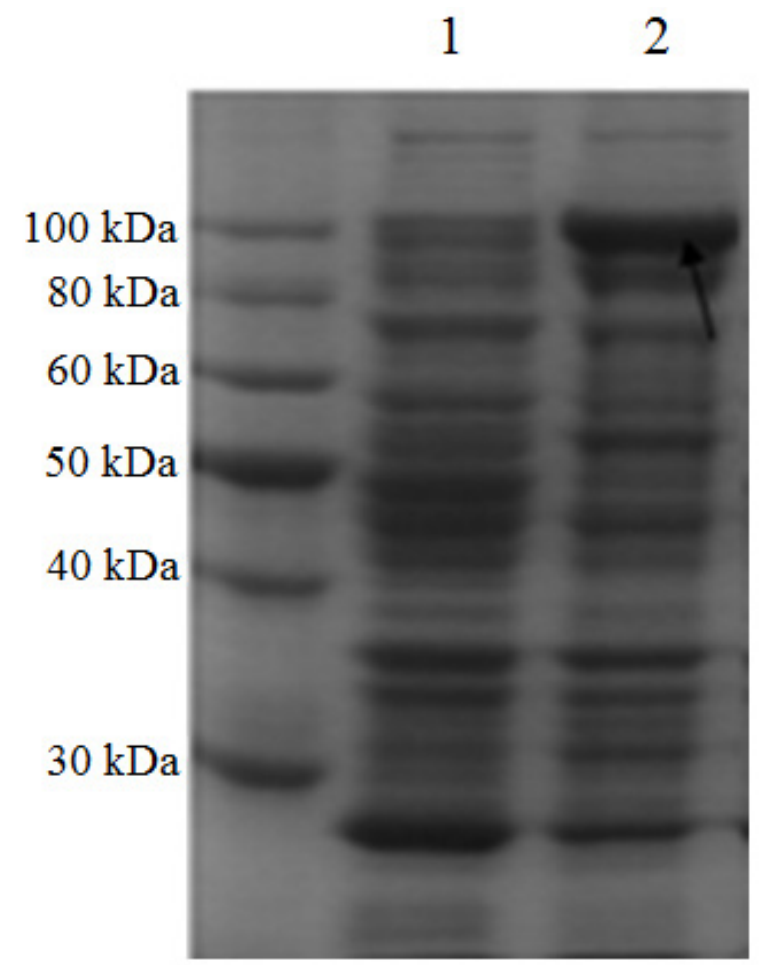

Figure 3. Exogenous protein expression of GmMEKK in Escherichia coli. Lanes 1 and 2 are without and with IPTG, respectively.

\section{Expression of GmMEKK in different soybean tissues}

Expression pattern analyses of GmMEKK in soybean revealed highest expression levels in leaves, followed by roots, stems, flowers, pods, and no expression in seeds (Figure 4). These data suggest that $G m M E K K$ may have important biological functions in soybeans.

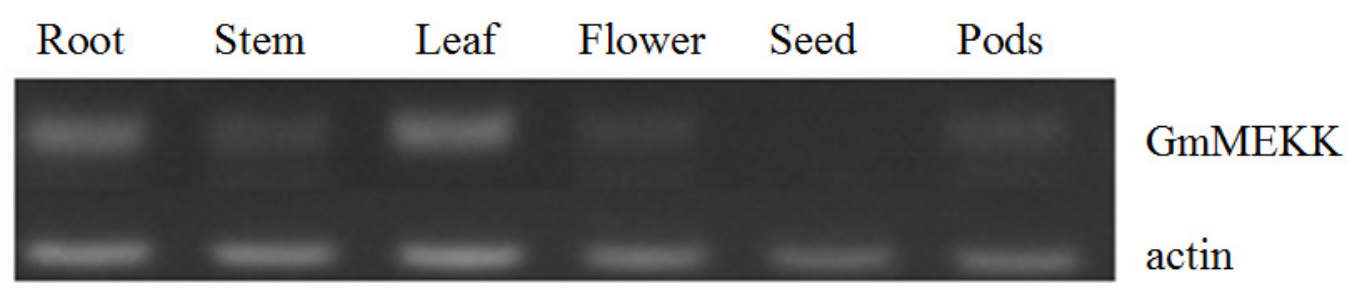

Figure 4. Expression patterns of GmMEKK in soybean tissues. 


\section{Expression of $G m M E K K$ under different abiotic stresses}

Relative expression levels of GmMEKK in roots increased upon exposure to low phosphorus levels with expression peaking on the sixth day, with levels 5 times greater than those of $0 \mathrm{~h}$. The levels gradually decreased to a minimum on the ninth day. Under similar conditions, the control group was relatively stable with the expression ranging from about 0.9 to 1.2 times. Under low nitrogen conditions, the relative expression levels of GmMEKK in roots initially increased and peaked on the ninth day, then gradually decreased, while the control group remained relatively stable. Under drought stress conditions, the relative expression levels of GmMEKK in roots dramatically increased from 10 to 12 times within $2 \mathrm{~h}$ (Figure 5).
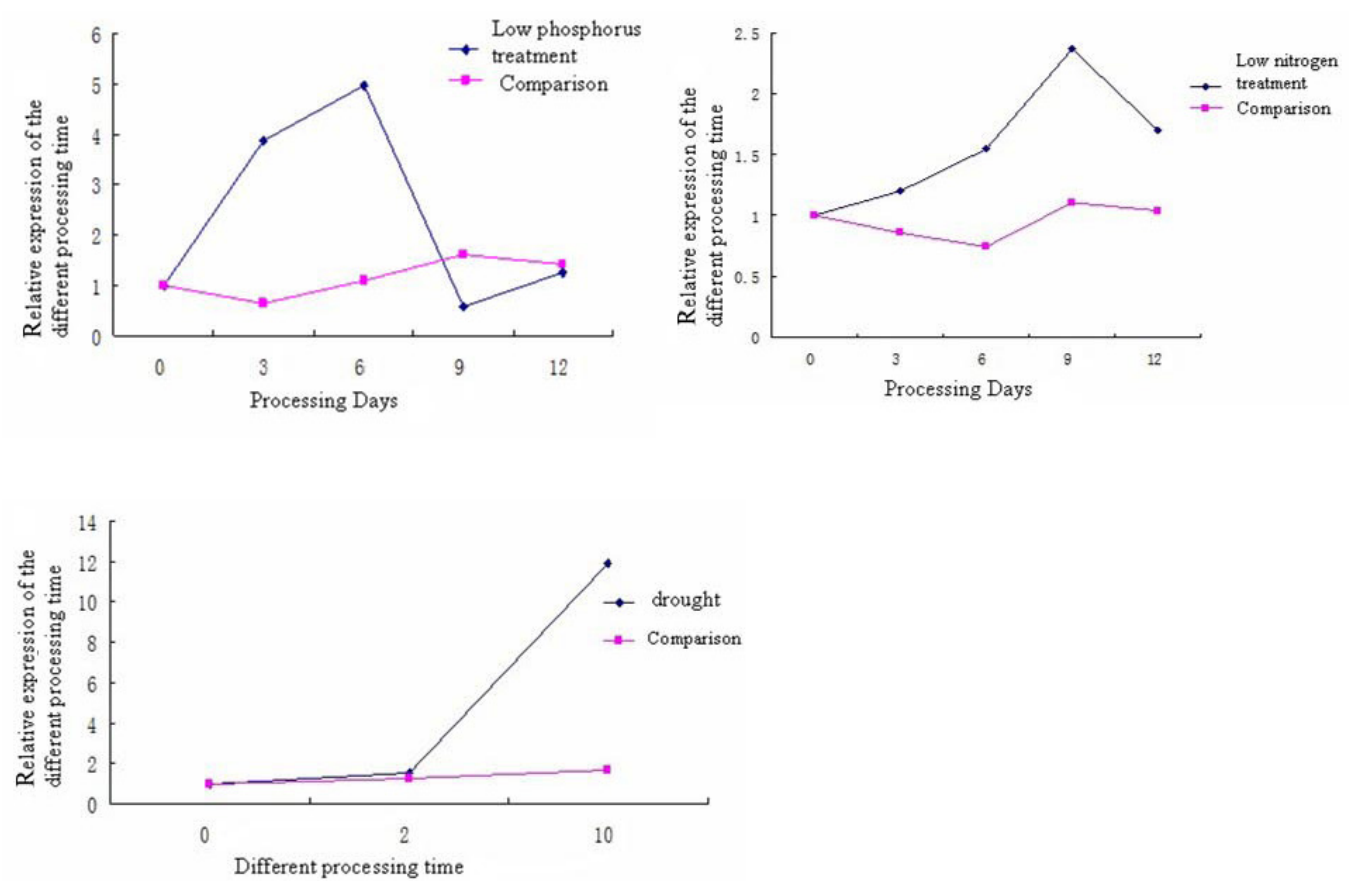

Figure 5. Expression levels of GmMEKK under different abiotic stresses.

\section{DISCUSSION}

Among MAPKs, the numbers of MAPKKKs are the most with more than 60 members cloned in Arabidopsis (Suarez-Rodriguez et al., 2007) and a dozen in mammalian cells (Mou and Chen, 2002). In this study, we cloned the GmMEKK and performed a protein-level homological analysis with MAPKs of plants such as alfalfa, cabbage-type rape, Arabidopsis, cultivated rice, sorghum, corn, canola, and Western balsam. We found that both their domains and sequences are homologous, evolutionarily conserved, and all share characteristics typical of MEKK1 (STE11/BCK1) protein kinases. Therefore, GmMEKK belong to MEKK1 (STE11/ BCK1) class of MAPKKK genes. Spatial gene expression patterns are often related to their biological functions (Gao et al., 2008). For example, upon activation, MAPKs that enter the 
nucleus can regulate gene expression through the activation of specific transcription factors, whereas cytoplasmic MAPKs will be responsive to outside stimulus by phophorylating the upstream proteins outside ostensive stimulus (Ning et al., 2010). As a result, understanding the subcellular localization of a protein can provide important information about its functions. As demonstrated by its subcellular localization, GmMEKK was found in the nucleus to serve in the upstream of the MAPK signaling cascade to receive signals conveyed by other upstream genes such as receptor protein kinase, protein kinase $\mathrm{C}$, and small $\mathrm{G}$ protein and is activated. Upon activation, GmMEKK catalyzes the phosphorylation of downstream genes, and initiates the MAPK signaling cascade. On the other hand, GmMEKK may regulate a subset of transcription factors required to maintain and control biochemical reactions in response to physiological conditions. However, these functions require further investigation.

In conditions of drought and nutrient deprivation stress, plants will undergo physiological and biochemical adaptive responses in an attempt to reduce and/or eliminate damages caused by these environmental challenges. These responses are caused by changes of cellular expression. At the level of transcription, many genes involved in the stress response will be up- or down-regulated in response to biotic and abiotic stresses. Under drought and low phosphorus and nitrogen conditions, GmMEKK expression levels increased to varying degrees indicating that $G m M E K K$ may play a role in the stress response.

This study lays the foundation for in-depth research focused on the functional mechanisms of MAPK kinase cascades in soybean. The separation and identification of MAPKs specifically related to drought and nutrient deprivation is critical in confirming the molecular reactions and signal transmission of plants under conditions of environmental stress. Therefore, the identification of key factors closely related to plant stress resistance may help us further our understanding of the plant stress response and promote their resistance through integrated transgenic approaches.

\section{ACKNOWLEDGMENTS}

Research supported by the Key National Key Technology Support Program (\#2011BAD35B06) and the National Key Technology R\&D Program (\#2013AA102602-3).

\section{REFERENCES}

Gao MH, Liu JM and Zhang YL (2008). MEKK1, MKK1/MKK2 and MPK4 function together in a mitogen-activated protein kinase cascade to regulate innate immunity in plants. Cell Res. 18: 1190-1198.

Hirofumi N, Hanka S, Adam S, Zárský V, et al. (2006). A mitogen-activated protein kinase kinase kinase mediates reaction oxygen species homeostasis in Arabidopsis. J. Biol. Chem. 281: 38697-38704.

Mou JY and Chen XG (2002). Mitogen-activated protein kinase (MAPK) signal transduction pathway. Chin. Bull. Life Sci. 14: 208-211.

Ning J, Li XH and Xiong LZ (2010). A Raf-like MAPKKK gene DSM1 mediates drought resistance through reactive oxygen species scavenging in rice. Plant Physiol. 152: 876-890.

Suarez-Rodriguez MC, Adams-Phillips L, Liu YD, Wang H, et al. (2007). MEKK1 is required for flg22-induced MPK4 activation in Arabidopsis plants. Plant Physiol. 143: 661-669.

Xu BQ, Li JX and Gong XG (2005). Mitogen-activated protein kinase kinase. J. Cell Biol. Zhi 27: 387-390. 